\title{
Unpolarized azimuthal asymmetries in SIDIS at COMPASS
}

\section{Giulio Sbrizzai}

Trieste University and INFN

E-mail: giulio.sbrizzai@ts.infn.it

The study of the spin structure of the nucleon and of the effects due to the quarks transverse momentum are part of the scientific program of COMPASS, a fixed target experiment at the CERN SPS. The azimuthal modulations which appear in the cross-section of SIDIS off unpolarised targets give insight on the intrinsic momentum structure of the nucleon and on the possible correlation between transverse spin and transverse momentum of the quarks. We present the results for the amplitudes of the $\cos (\phi), \cos (2 \phi)$, and $\sin (\phi)$ modulatuions (where $\phi$ is the azimuthal hadron angle in the gamma-nucleon system) obtained from the COMPASS data collected with a $160 \mathrm{GeV} / \mathrm{c}$ positive muon beam impinging on a deuteron target. The amplitudes are measured separately for positive and negative hadrons, and the results on the dependence of the relevant kinematic variables obtained after a multi dimensional analysis are also presented.

XXI International Workshop on Deep-Inelastic Scattering and Related Subject-DIS2013, 22-26 April 2013

Marseilles,France 


\section{Introduction}

The investigation of the transverse spin and transverse momentum structure of the nucleon is one of the main topics of the COMPASS experiment. It is carried on by studying the azimuthal distributions of the hadrons produced in deep inelastic scattering (DIS) of $160 \mathrm{GeV} / \mathrm{c}$ positive muons off polarized and unpolarized nucleons.

All the independent azimuthal modulations expected to appear in the spin dependent SIDIS cross section can be measured at COMPASS from the same data. Their amplitudes are related both to the collinear Parton Distribution Functions (PDFs) and to the Transverse Momentum Dependent (TMD) PDFs describing the inner structure of the polarized nucleon.

The measurement of the spin-averaged hadron azimuthal asymmetries gives important information on the quark transverse momentum and on the higher-twist effects. Three modulation on the azimuthal angle of the hadron $\phi$ calculated in the Gamma Nucleon System are expected. The $\cos \phi$ asymmetry is mainly given by the intrinsic transverse momentum of the quark and it is a kinematic effect [1] (also known as "Cahn effect"). The TMD Boer-Mulders PDF [2], which correlates the quark transverse polarization and its intrinsic transverse momentum inside an unpolarized nucleon, appears at leading twist in the amplitude of the $\cos 2 \phi$ modulation. The same prediction on the change of sign from SIDIS to DY, as for the Sivers function, applies to the Boer-Mulders PDF [3]. The Cahn effect is also involved in this modulation, but at twist 4, which means suppressed by a factor $1 / Q^{2}$. The $\sin \phi$ modulation is due only to higher twist effects and its amplitude is proportional to the beam polarization.

\section{Spin-averaged asymmetries results}

The amplitudes of the $\cos \phi, \cos 2 \phi$ and $\sin \phi$ modulations extracted separately for positive and negative hadrons have been measured from the 2004 deuteron data [4]. The corresponding asymmetries are extracted binning the data in $x, z$ or $P_{T}^{h}$. The hadron azimuthal distributions calculated from the real data are corrected for the acceptance effects using Monte Carlo simulations of the apparatus. All the possible effects, the geometrical acceptance, the triggers' and detectors' inefficiencies and the interaction of the particles with the material of the spectrometer, are simulated. The same program as for the real data is used for the tracks reconstruction.

The results are shown in fig. 1 , for $\cos \phi$, and in fig. 3 , for $\cos 2 \phi$. Large signals and strong dependences on the kinamatical variables have been found. More studies have been done to investigate in more details the interesting kinematic dependencies found, and a multi dimensional analysis has been performed binning simultaneously the data in the 3 variables $x, z$ and $P_{T}^{h}$. The $\cos \phi$ and the $\cos 2 \phi$ modulations, shown for the forst time in [5], are shown here in fig. 2 and in fig. 4 respectively. The results for $\sin \phi$ are shown in fig. 5. The asymmetries are small, slightly positive for the positive hadrons and no further relevant trend had been found in the multi dimensional analysis.

Only the statistical errors appear in all the plots being the systematical errors evaluated to be roughly twice the statistical ones. 


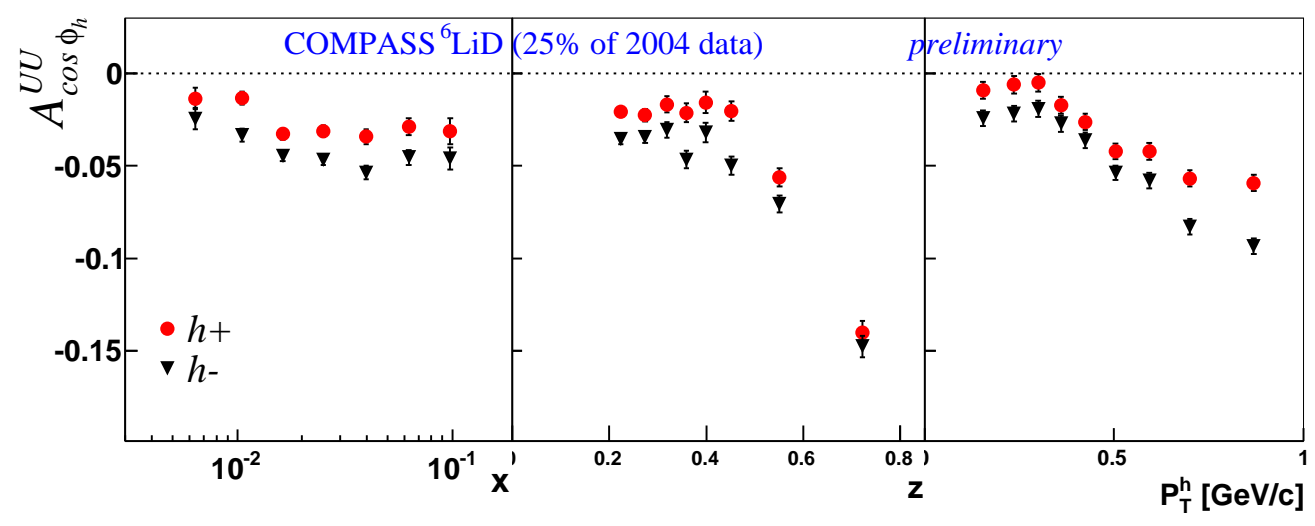

Figure 1: The $\cos \phi$ spin-averaged asymmetries as functions of $x, z$ or $P_{T}^{h}$, for the positive hadrons (red points) and negative hadrons (black points).

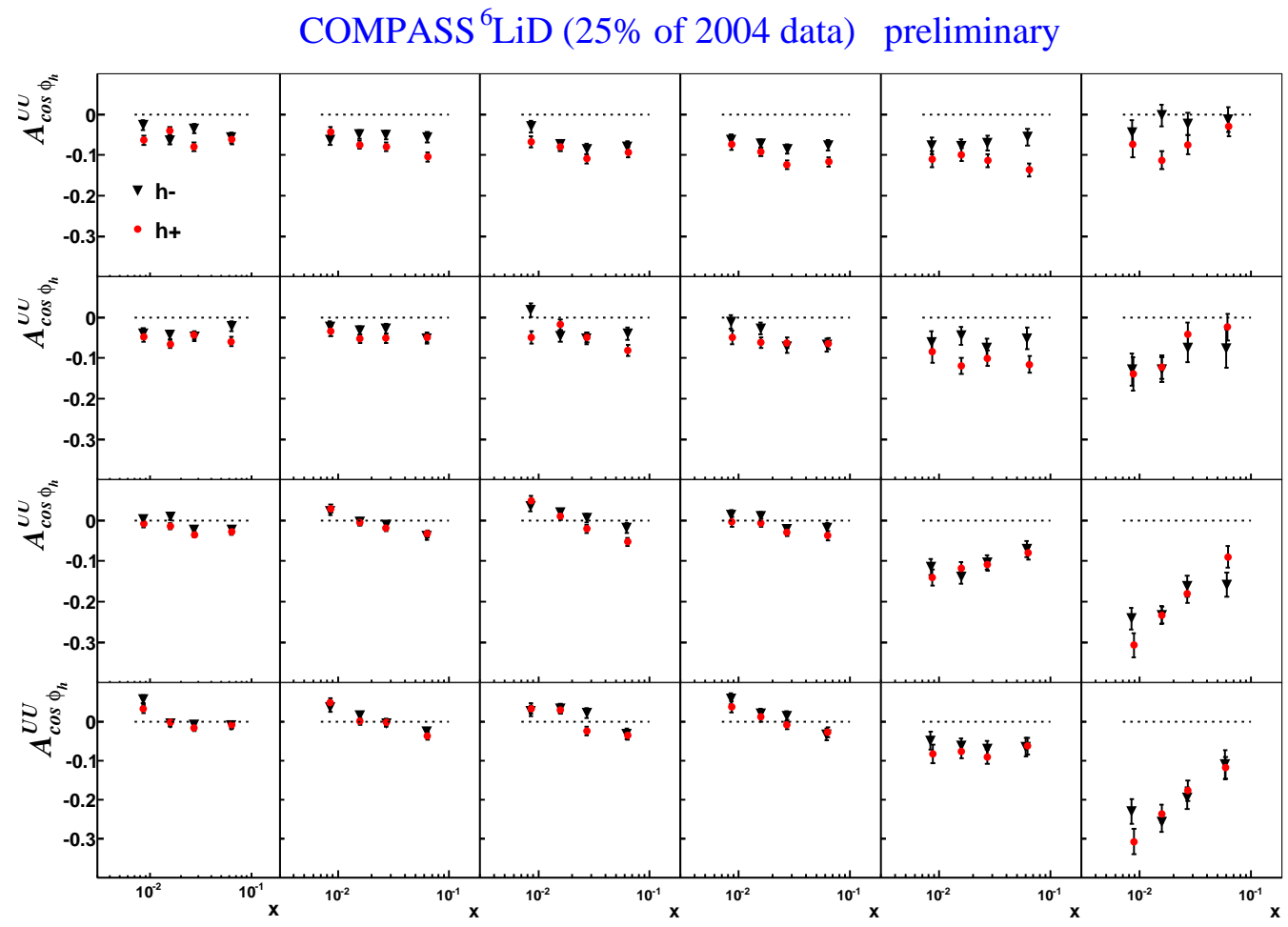

Figure 2: The $\cos \phi$ spin-averaged asymmetries as functions of $x$, extracted in different bins of $z$ (from left to right) and $P_{T}^{h}$ (from bottom to top), for the positive hadrons (red points) and negative hadrons (black triangles). 


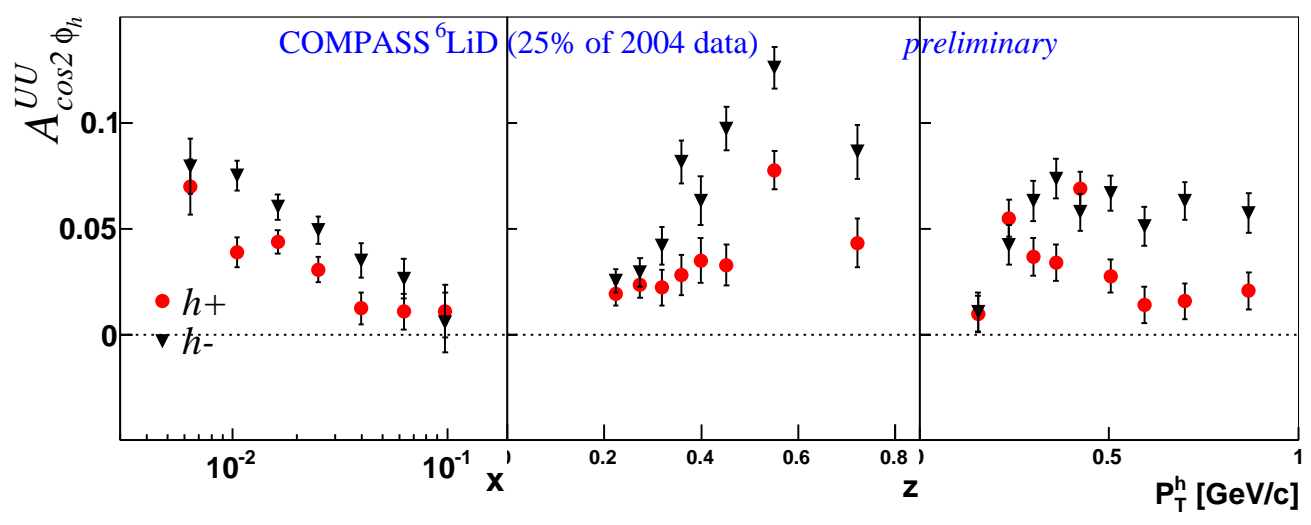

Figure 3: The $\cos 2 \phi$ spin-averaged asymmetries as functions of $x, z$ or $P_{T}^{h}$, for the positive hadrons (red points) and negative hadrons (black points).

COMPASS ${ }^{6} \mathrm{LiD}(25 \%$ of 2004 data) preliminary

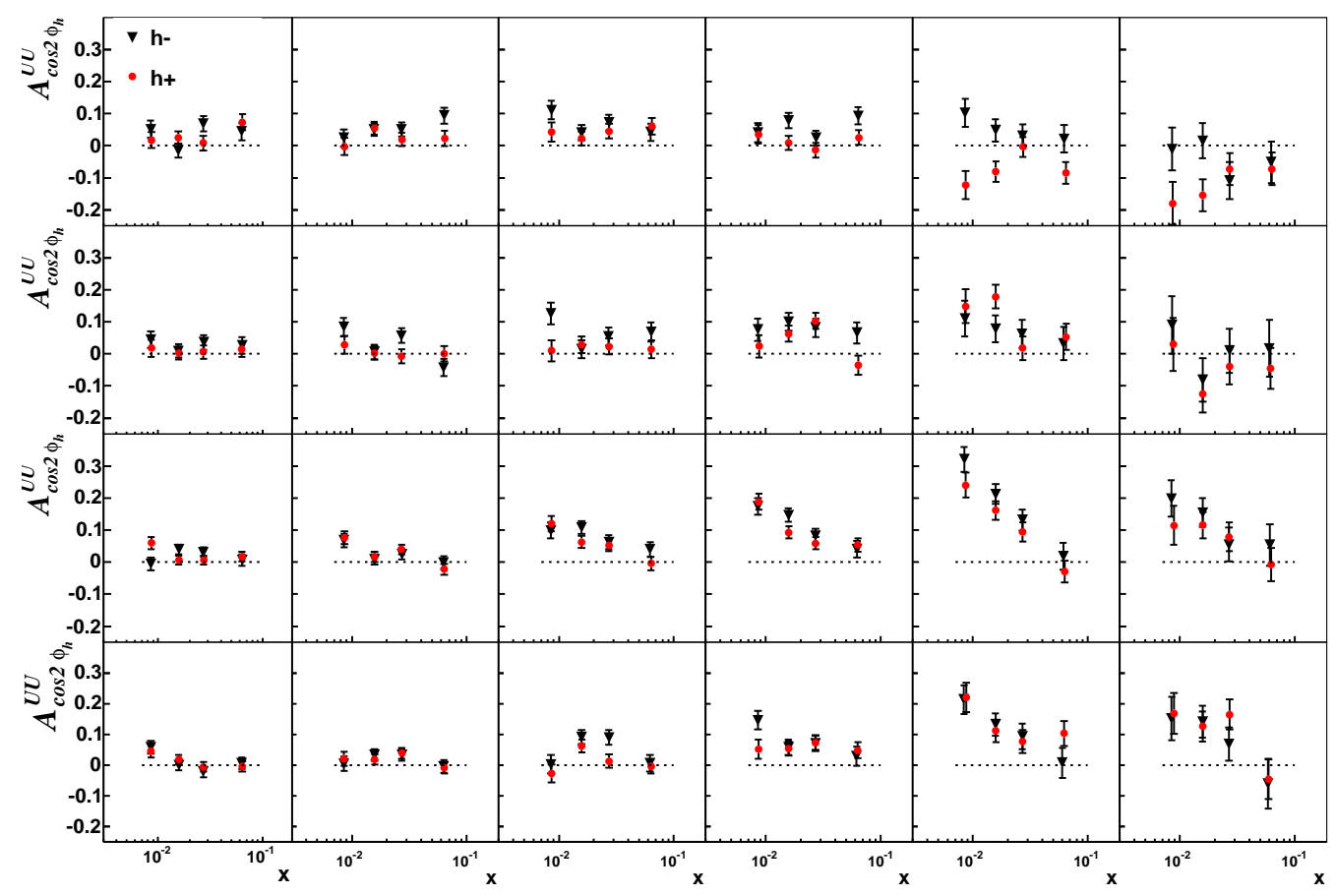

Figure 4: The $\cos 2 \phi$ spin-averaged asymmetries as functions of $x$, extracted in different bins of $z$ (from left to right) and $P_{T}^{h}$ (from bottom to top), for the positive hadrons (red points) and negative hadrons (black triangles). 


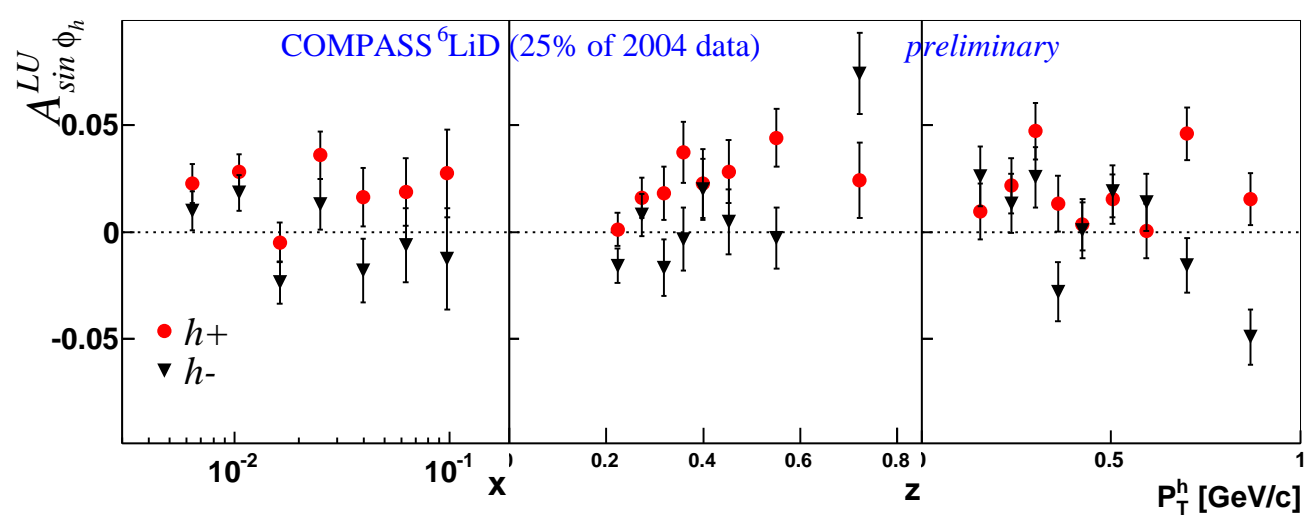

Figure 5: The $\sin \phi$ spin-averaged asymmetries as functions of $x, z$ or $P_{T}^{h}$, for the positive hadrons (red points) and negative hadrons (black points).

\section{Conclusions}

The kinematic dependencies of the SIDIS spin-averaged azimuthal asymmetries have been studied in the three-dimensional $x-z-P_{T}^{h}$ grid. The strong $z$ dependence already found in the onedimensional analysis seems to come from the small $x$ and small $P_{T}^{h}$ region. It is also interesting to note that the $x$ trend clearly change at large $z$ values $(z \gtrsim 0.5)$. These results provide an interesting input for theory and their interpretation must take into account the different $Q^{2}$ region covered by the different bins.

\section{References}

[1] R.N.Cahn, Phys. Lett. B 78 269, (1978).

[2] D.Boer, P.J.Mulders Phys. Rev. D 57 (1998) 5780-5786.

[3] J.C. Collins Phys. Lett. B536 (2002) 43 [hep-ph/ 0204004$]$

[4] G. Sbrizzai, in proceedings of SPIN2010 Conference, J. Phys.: Conf. Ser. 295012043

[5] G. Sbrizzai, in proceedings of SPIN2012 Conference 